\title{
Biomechanics of Degenerative Spinal Disorders
}

\author{
Justin A. Iorio ${ }^{1}$, Andre M. Jakoi ${ }^{2}$, Anuj Singla ${ }^{3}$ \\ ${ }^{1}$ Department of Orthopedic Surgery, Hospital for Special Surgery, New York, NY, USA \\ ${ }^{2}$ Department of Orthopedic Surgery Spine Center, University of Southern California, Los Angeles, CA, USA \\ ${ }^{3}$ Department of Orthopedic Surgery, University of Virginia, Charlottesville, VA, USA
}

\begin{abstract}
The spine has several important functions including load transmission, permission of limited motion, and protection of the spinal cord. The vertebrae form functional spinal units, which represent the smallest segment that has characteristics of the entire spinal column. Discs and paired facet joints within each functional unit form a three-joint complex between which loads are transmitted. Surrounding the spinal motion segment are ligaments, composed of elastin and collagen, and joint capsules which restrict motion to within normal limits. Ligaments have variable strengths and act via different lever arm lengths to contribute to spinal stability. As a consequence of the longer moment arm from the spinous process to the instantaneous axis of rotation, inherently weaker ligaments (interspinous and supraspinous) are able to provide resistance to excessive flexion. Degenerative processes of the spine are a normal result of aging and occur on a spectrum. During the second decade of life, the intervertebral disc demonstrates histologic evidence of nucleus pulposus degradation caused by reduced end plate blood supply. As disc height decreases, the functional unit is capable of an increased range of axial rotation which subjects the posterior facet capsules to greater mechanical loads. A concurrent change in load transmission across the end plates and translation of the instantaneous axis of rotation further increase the degenerative processes at adjacent structures. The behavior of the functional unit is impacted by these processes and is reflected by changes in the stressstrain relationship. Back pain and other clinical symptoms may occur as a result of the biomechanical alterations of degeneration.
\end{abstract}

Keywords: Biomechanics; Degenerative; Spinal disorders

\section{Introduction}

The spine is a complex structure that provides protection and support to the spinal cord in different positions and postures. The bony and soft-tissue anatomy are intimately related and function within a spinal motion segment to provide stability and movement about three axes, which form a Cartesian coordinate system. Within this coordinate system, translation and rotation can occur around each axis. Normal aging of the spine affects the spinal elements and, potentially, causes a sufficient degree of degeneration to alter the motion segment. Degeneration of the intervertebral disc space results in a reduction of disc height and abnormal load transmission across the vertebral end plates and paired facet joints, which play a pivotal role in the three-joint spinal complex. As the biomechanical properties of the spine change, there is also an alteration in the stress-strain relationship as well as translation of the instantaneous axis of rotation (IAR) from its usual position [1]. This complex process of degeneration moves across stages including dysfunction, instability, and stabilization [2] and may ultimately produce low back pain and other clinical symptoms. Biomechanics affords a means of characterizing and assessing the status of the spine both

Received Aug 27, 2015; Revised Aug 31, 2015; Accepted Sep 5, 2015

Corresponding author: Anuj Singla

Department of Orthopedics, University of Virginia, 400 Ray C Hunt Drive, Charlottesville, VA 22903, USA

Tel: +1-434-243-0291, Fax: +1-434-243-0290, E-mail: As7bv@virginia.edu 
precisely and quantitatively. Benefits of an improved understanding of biomechanics of normal and degenerative spinal conditions are the ability to counsel patients, treat pathological processes, and determine the effect of both medical and surgical treatment on spinal mechanics and, potentially, clinical outcomes. Understanding the biomechanical consequences of degeneration is imperative for the treatment of spinal disorders, regardless of etiology. In this review, we discuss key concepts of spinal anatomy and degenerative processes of the spine.

\section{Anatomy}

Thirty-three vertebrae comprise the spinal column: 7 cervical, 12 thoracic, 5 lumbar, 5 sacral, and 4 coccygeal bones. Functionally, the spinal column transmits loads, permits limited motion, and protects the spinal cord. The range of normal spinal alignment is dependent upon the region of the spine. In the coronal plane, the normal spine has a neutral curvature. In the sagittal plane, the cervical and lumbar segments are lordotic whereas the thoracic and sacral regions are kyphotic. Regional kyphosis or lordosis are evolutionary responses to an upright stance in the bipedal human and serve to balance the occiput over the pelvis in an energy efficient manner [3,4]. However, vertebral bodies within a spinal segment do not evenly distribute alignment. Cervical spine lordosis is greatest at C1-2 (75\%) while C4-7 contributes very little to overall lordosis [5]. Similarly, L4-5 and L5-S1 provide about twothirds of lumbar lordosis [6]. Sagittal alignment can be measured by dropping a plumb line from the $\mathrm{C} 7$ vertebral body to the lumbosacral junction and, when within normal limits, permits balanced posture, minimal energy expenditure, and appropriate tension of perispinal ligaments [7]. The intimate relationships between global balance and both clinical outcomes [8,9] and biomechanics [10] have been reported. As sagittal imbalance increases, defined as a plumb line that fails to fall between the sacrum and femoral heads, the pelvis undergoes retroversion in relation to the feet. The change in pelvic positioning maintains a fixed gravity line-heel offset, which preserves a center of force near the feet and permits standing balance [10].

The functional spinal unit (FSU) or spinal motion segment is the smallest segment that represents the characteristics of the entire spinal column. It consists of two vertebrae, the intervertebral disc, zygaphophyseal (facet) joints, and supporting ligaments (ligamentum flavum, su- praspinous, interspinous, anterior longitudinal, and posterior longitudinal). The disc and paired facet joints at each level therefore form a three-joint complex between which loads are transmitted [11]. The intervertebral disc functions to transmit loads between adjacent vertebrae and permit motion. As such, it carries and distributes forces to which the trunk is subjected [12]. Each motion segment has an IAR, which is a dynamic point about which the FSU rotates and is dependent upon spinal alignment and forces acting on the spine. There are 12 potential movements about the IAR due to rotation around the three axes $(x, y$, and $z)$ that pass through the center of rotation. The IAR is not constant; for example, during flexion-extension at $\mathrm{C} 0-\mathrm{C} 1$ the IAR passes through the center of the mastoid processes whereas during lateral bending the IAR is located $2 \mathrm{~cm}$ above the dens [13]. The IAR in the lumbar spine is similarly dependent upon position. The IAR is located in the anterior disc in flexion, lateral aspect of the disc with contralateral side-bending, and in the posterior annulus during axial rotation $[14,15]$. Surgical intervention, trauma, and degenerative processes affect the position of the IAR.

Soft tissues about the spine also play a tremendous role in flexibility and mobility. Below the subaxial spine, there are seven ligaments that play a paramount role in maintaining physiologic motion. Ligaments, composed of elastin and collagen [16], and joint capsules restrict motion to within normal limits. The ligaments have variable strengths, but weaker ligaments such as the interspinous and supraspinous contribute greatly to spinal stability by providing resistance to flexion via a long moment arm from the spinous process to the IAR [17]. The ligamentum flavum, in contrast, is a pair of broad ligaments that provides compression at the disc space by maintaining resting tension as it courses along the ventral laminae [18]. Spinal motion is also influenced by intervertebral discs and the synovial facet joints, which consist of sliding cartilaginous surfaces. Although facet joints bear only approximately $10 \%$ of the load during axial loading, they are subjected to a nearly threefold increase in load-bearing during extension and fivefold increase in ventral shear forces during flexion [17]. Increases in postural load-bearing coupled with decreasing elastin and increasing collagen concentration in facet capsules with aging prohibits viscoelastic materials from returning to their elastic zone. The result is stretching of soft tissues, increasing motion between interfaces, and adaptations of bony architecture to provide 
mechanical support.

\section{Disc Degeneration}

Degenerative disc disease begins early in life and precedes the development of facet joint changes. A histologic study of age-related changes in the human lumbar disc revealed reduced end plate blood supply that resulted in the breakdown of the nucleus pulposus by the second decade of life [19]. The significance of this can be understood by considering the function and response of the healthy disc during normal movements. During normal motion, the disc deforms predictably: the annulus bulges on the side of compression and is under tension on the opposite side. Under compression, load transmission between two vertebrae is via the intervertebral disc, which is composed of type II collagen. Resultant pressures within the pulposus are transferred to the annulus. Horst and Brinckmann [20] performed a cadaveric study of axial stress distribution by implanting pressure transducers within thoracic and lumbar intervertebral discs. Their results showed that stress distribution is dependent upon the status of the disc and differs between thoracic and lumbar spinal segments. Healthy lumbar discs distributed stress evenly across the endplates in compression and eccentric loading (increasing end-plate inclination). The same uniform stress distribution occurred in degenerative discs under axial compression. Eccentric loading of degenerative discs, in contrast, produced asymmetric force distribution which increased with severity of degeneration and increasing end-plate inclination. Thoracic discs, unlike lumbar discs, displayed uniform stress distribution under compression only. Eccentric loading caused asymmetric stress distribution in both healthy and degenerative discs. The transition zone between discs with thoracic and lumbar properties occurs at T11 and T12 because these vertebrae are more mobile than the mid-thoracic spine.

Other studies have also reported on differences in the responses of discs to applied loads between spinal segments. Kulak et al. [21] studied the nonlinear, rateindependent behavior of intervertebral discs with a finite element model to specifically investigate the elastic zone of the stress-strain curve. The annulus fibrosis in the lumbar spine responded nonlinearly to applied forces because of the behavior of the collagen fibers [21], which in a prior study was shown to have an elastic component of collagen that was proportional to the square of the strain [22]. In the thoracic spine, the elastic response of the annulus was linear and hoop stresses were less dominant because annular fibers were compressed near the endplates due to greater constraint by the endplates [21]. Stress distribution also differed between lumbar and thoracic segments. In the lumbar spine, the fiber stress is tensile throughout the annulus and increases from the inner to the outer boundary of the annulus; the maximum fiber stress is therefore at the periphery of the disc. Lumbar collagen fibers are under tension because of their greater relative disc height to width ratio compared to thoracic discs. Thoracic annuli were shown to have lower fiber stress values through the mid-region and were often in compression above the midplane. Load-deflection curves from the analysis found that intervertebral discs became stiffer (i.e., less displacement for a given load) as the spine was ascended from L3-4 to T5-6 and less stiff from T5-6 to T1-2 [21,23]. Enucleation of the lumbar disc decreased stiffness by twofold, thus demonstrating the importance of the pulposus under compression [21]. A cadaveric study of lumbar motion segments reported the effect of disc degeneration on the mechanical instability of the spine [1]. Using a spine simulator with six degrees of freedom and unconstrained, cyclical loading, lumbar discs with increasing stages of degeneration were found to have erratic excursions of the IAR, vertical translation of the center of rotation, and abnormal load distribution across the disc space with lower pressures in the region of the nucleus and pressure spikes in the annular region. Osteophytes develop as the annulus is distorted and pulls from its bony attachments. The result is an unstable FSU and, potentially, low back pain.

Biomechanical changes of the unstable FSU and fused FSU are reflected in their respective stress-strain curves (Fig. 1). Normally, the stress-strain curve of a healthy disc consists of a neutral zone and an elastic zone which have

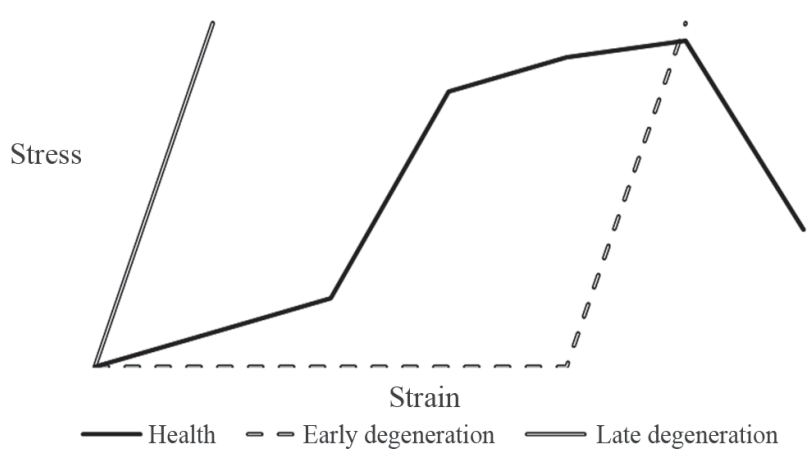

Fig. 1. Stress/strain relationship of healthy vs. degenerative disc. 
different responses to a given load. Under initial loading, the disc undergoes a large amount of deformation compared to the applied load because of disc bulging (neutral zone). With increasing loads, the fibers of the annulus restrict the bulging of the pulposus in the elastic zone such that less deformation occurs with increasing compression. Unstable spinal segments have larger neutral zones because of ligament laxity and loss of disc height, and the annulus degenerates and loses its ability to provide hoop stress compression of the nucleus $[1,17]$. This results in dorsal translation of the IAR and overloading of the facet joints. In contrast, the latter stages of disc degeneration produce an entirely different morphology of the stressstrain curve. The inner annulus expands and the boundary between the nucleus pulposus and annulus fibrosus becomes indistinguishable. The fibrotic disc loses its fluidlike properties and behaves like a solid material [20]. The fused spinal segment exhibits a stress-strain curve similar to that of a stiffer, more brittle material: the slope of the elastic zone is steeper than the normal FSU, representing the incompressibility of the fused segment and requirement for large loads to produce little deformation. In the lumbar spine, spinal instability does not refer to an abnormally increased range of motion; more accurately, range of motion increases in the early stages but diminishes in later stages of degeneration $[24,25]$.

\section{Facet Joint Degeneration}

The facet joints are the only true synovial joints of the spine. The facet is an articulation between the mediallyfacing superior articular process of the lower vertebra and a smaller, laterally-facing inferior articular process originating from the superior vertebra. Menisci within the articulation function to maintain smooth gliding surfaces and fill voids that develop between incongruent facet joints [26]. Capsules surrounding facet joints have important functions of restricting rotation and dorsal displacement during extension $[27,28]$. Facet joint orientation determines the plane of motion and varies as one descends the spinal column. Upper cervical spine superior articular facets face posteromedially and transition to face posterolaterally between $\mathrm{C} 4$ and T1, with the C5-6 level representing the most common transition point [29]. The facet joints are oriented approximately $0^{\circ}$ in the coronal plane and $45^{\circ}$ in the sagittal plane from C3-7. The spatial orientation of the facets explains the coupled motion of lateral bending and axial rotation in this region [13]. During lateral bending, the ipsilateral inferior articular process of the cephalad vertebra descends along the facet in the sagittal plane and simultaneously translates posteriorly. The contralateral inferior process ascends the inclined facet and moves anteriorly. Thoracic spine facet joints are positioned in about $20^{\circ}$ of coronal and $55^{\circ}$ of sagittal orientation and exhibit the same coupling behavior as the cervical spine. Lower thoracic (T9-12) facets typically resemble lumbar facet joints, however. The result is that the upper and middle thoracic spine allow more axial rotation. Lumbar spine facet joints exhibit the greatest coronal and sagittal orientation: $50^{\circ}$ and $90^{\circ}$, respectively. Sagittal facet orientation restricts rotational movements but has less ability in preventing flexion-extension and translation. Thus, the lumbar facets are predisposed to listhesis as the spine degenerates. Further, the sagittal orientation of lumbar facets increases with normal aging regardless of the presence of the spondylolisthesis (Fig. 2) [30]. Coupling of motion in the lumbar spine is such that flexionextension is couple with both axial rotation and lateral bending.

Biomechanically, facet joints bear loads in the posterior column, restrict axial rotation, and control flexion-extension motions. In flexion, facet joints prohibit excessive anterior translation of vertebral bodies. Despite their important role in restricting supraphysiologic motion, the forces acting upon the joints are not without consequence. At terminal flexion and extension, the joints gape and "edge load", producing increased contact pressure on the lower edge of the inferior articular process and superior edge of the opposing articular process $[11,31,32]$, and in the upright position the facets are exposed to continuous shear forces most notably at L5-S1 [33]. One potential reason for the development of coronally-oriented lumbar facets is to reduce shear forces [34]. In the healthy FSU, facet joints bear up to $25 \%$ of the load transmitted across the 3 -joint complex; however, the load nearly doubles across facet joints in degenerative conditions depending upon body position [31,35]. Cartilage degeneration of the facet joints results in bony erosions and eventual sclerosis of the subchondral bone in a process similar to other joints in the body. In a cadaveric study of lumbar facet joints, cartilage degeneration was most pronounced at the articular margins and generally well preserved in the central region [36]. In the same study, osteophytes were most commonly found on the lateral margin of the superior articular pro- 

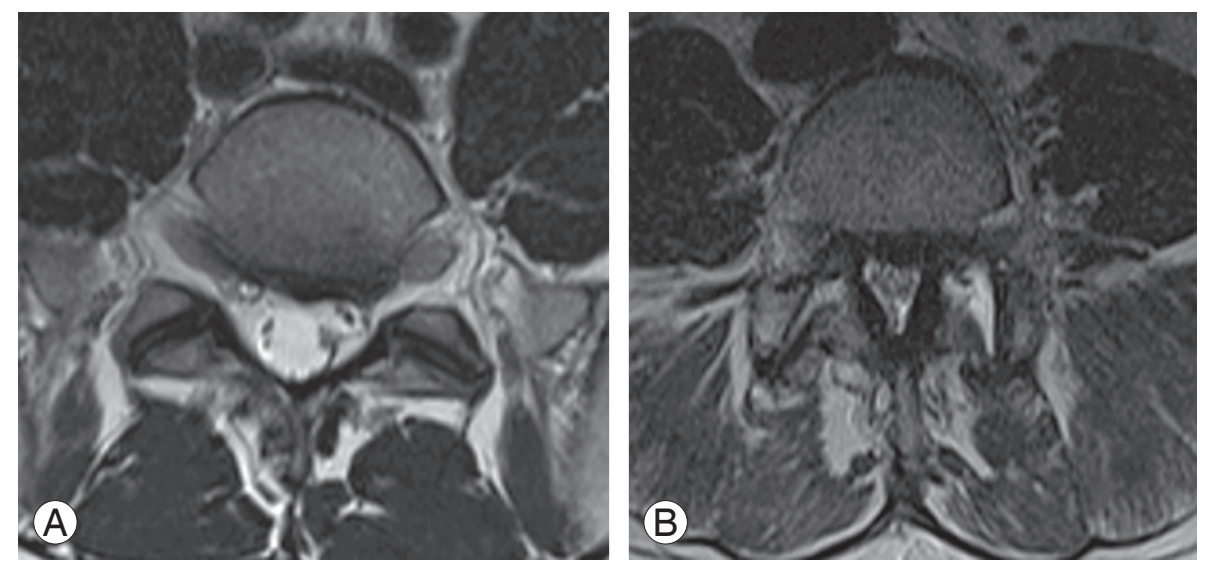

Fig.2. Facet joint degenerative changes. (A) Normal sagittal orientation of lumbar facet joints. (B) Ligamentum flavum thickening, facet hypertrophy and coronal orientation of facet joints.

cess. At this location, the capsule attaches to the facet and as the spine rotates axially the posterior aspect of the contralateral joint capsule is subjected to tensile forces.

As disc degeneration occurs and disc height decreases, the increased range of axial rotation subjects the posterior capsule to greater mechanical loads. Boszczyk et al. [37] confirmed in an immunohistochemical analysis of facet capsules that the greater mechanical demand of degenerative spinal segments produces fibrocartilaginous metaplasia (primarily at the entheses), capsular hypertrophy, and bone spur formation. A potential result of facet osteoarthritis is low back pain, which is a response transmitted from the medial branches of the primary dorsal rami. Nerve impingement may also occur as the facet hypertrophies and impedes on the neural foramen bordered by the superior articular process, pars interarticularis, and vertebral body.

\section{Adjacent Segment Disease}

Cervical and lumbar pathology which requires stabilization may initially address the problem of instability but can ultimately lead to other long-term consequences such as adjacent segment disease. Adjacent segment disease was first noted in the lumbar spine following fusion surgery [38] but follow-up studies eventually led to the recognition and awareness of this disease in the cervical spine as well $[39,40]$. Defined as postarthrodesis adjacent level pathology by Hilibrand and Robbins [39], the term adjacent segment degeneration describes radiographic changes observed at levels next to the previously fused segment or segments. It is important to note that this de- generation may not correlate with clinical symptoms. In contrast, adjacent segment disease refers to the development of new myelopathy or radiculopathy referable to a motion segment adjacent to the site of a previous arthrodesis [40].

From a mechanical perspective, it is a reasonable hypothesis that after motion is eliminated from a spinal level, to achieve the same range of motion, the remaining spinal levels would be required to take on a greater load. This was subsequently demonstrated in several studies involving surgical arthrodesis of the spine. Surgical arthrodesis has been proven to influence mechanical properties of intervertebral disks next to a fused level in the cervical and lumbar spine [41,42]. Studies have shown a pervasiveness of hypermobility and increased stress in the adjacent segments [41,42]. Maiman et al. [43] calculated increased internal stress responses at the adjacent segments, thereby reflecting changes in load sharing after fusion. Eck et al. [41] measured intradiscal pressure at adjacent levels following fusion in a cadaver model and found a $45 \%$ to $73 \%$ increase in intradiscal pressure, as well as a concomitant increase in segmental motion with flexion. Matsunaga et al. [44] analyzed the change in intervertebral disk strain distribution following anterior cervical discectomy with fusion in 96 patients by assessing their lateral dynamic radiographs preoperatively and postoperatively. At an average of 1 year following two- or three-level fusion, the authors observed a $20 \%$ increase in shear strain of adjacent segments. Ultimately, the increase in mobility and intradiscal pressure at levels adjacent to fusion causes an increase in internal stress at these levels. It has been shown that this increase in motion and intradiscal 
pressure is directly related to the number of levels fused $[45,46]$. Biomechanical studies therefore suggest that fusion causes increased stress and strain on the neighboring motion segments. This phenomenon can potentially contribute to accelerated degeneration for the patient and may ultimately lead to disease.

\section{Conclusions}

Degenerative changes affect bony and soft tissue structures of the spine and may ultimately result in modifications of the spinal motion segment and IAR. Normal age-related changes, despite representing expected processes on the continuum of aging, have the potential of producing mechanical and clinical symptoms because of the complex interrelations between all parts of the FSU. Loss of the disc height affects load transmission across end plates, facet joints, motion between vertebral bodies, the stress-strain relationship, and the IAR, which further increases loading of adjacent structures. In postsurgical cases, stress distribution to adjacent levels may accelerate degenerative processes. Age-related processes represent normal anatomic changes and a thorough understanding will enable the care provider to deliver treatments and patient education for optimal management.

\section{Conflict of Interest}

No potential conflict of interest relevant to this article was reported.

\section{References}

1. Sengupta DK, Fan H. The basis of mechanical instability in degenerative disc disease: a cadaveric study of abnormal motion versus load distribution. Spine (Phila Pa 1976) 2014;39:1032-43.

2. Kirkaldy-Willis WH, Farfan HF. Instability of the lumbar spine. Clin Orthop Relat Res 1982;(165):11023.

3. Schwab F, Lafage V, Boyce R, Skalli W, Farcy JP. Gravity line analysis in adult volunteers: age-related correlation with spinal parameters, pelvic parameters, and foot position. Spine (Phila Pa 1976) 2006;31:E959-67.

4. Berthonnaud E, Dimnet J, Roussouly P, Labelle H. Analysis of the sagittal balance of the spine and pelvis using shape and orientation parameters. J Spinal
Disord Tech 2005;18:40-7.

5. Hardacker JW, Shuford RF, Capicotto PN, Pryor PW. Radiographic standing cervical segmental alignment in adult volunteers without neck symptoms. Spine (Phila Pa 1976) 1997;22:1472-80.

6. Bernhardt M, Bridwell KH. Segmental analysis of the sagittal plane alignment of the normal thoracic and lumbar spines and thoracolumbar junction. Spine (Phila Pa 1976) 1989;14:717-21.

7. Schwab F, Patel A, Ungar B, Farcy JP, Lafage V. Adult spinal deformity-postoperative standing imbalance: how much can you tolerate? An overview of key parameters in assessing alignment and planning corrective surgery. Spine (Phila Pa 1976) 2010;35:2224-31.

8. Glassman SD, Bridwell K, Dimar JR, Horton W, Berven S, Schwab F. The impact of positive sagittal balance in adult spinal deformity. Spine (Phila Pa 1976) 2005;30:2024-9.

9. Glassman SD, Berven S, Bridwell K, Horton W, Dimar JR. Correlation of radiographic parameters and clinical symptoms in adult scoliosis. Spine (Phila Pa 1976) 2005;30:682-8.

10. Lafage V, Schwab F, Skalli W, et al. Standing balance and sagittal plane spinal deformity: analysis of spinopelvic and gravity line parameters. Spine (Phila $\mathrm{Pa}$ 1976) 2008;33:1572-8.

11. Adams MA, Hutton WC. The mechanical function of the lumbar apophyseal joints. Spine (Phila Pa 1976) 1983;8:327-30.

12. Hirsch C. The reaction of intervertebral discs to compression forces. J Bone Joint Surg Am 1955;37:118896.

13. Herkowitz HN, Rothman RH, Simeone FA. Rothman-Simeone, the spine. 5th ed. Philadelphia: Saunders Elsevier; 2006.

14. Cossette JW, Farfan HF, Robertson GH, Wells RV. The instantaneous center of rotation of the third lumbar intervertebral joint. J Biomech 1971;4:149-53.

15. Rolander SD. Motion of the lumbar spine with special reference to the stabilizing effect of posterior fusion: an experimental study on autopsy specimens. Acta Orthop Scand 1966:Suppl 90:1-144.

16. White AA 3rd, Panjabi MM. The basic kinematics of the human spine: a review of past and current knowledge. Spine (Phila Pa 1976) 1978;3:12-20.

17. Benzel EC. Spine surgery techniques, complication avoidance, and management (expert consult-online). 
London: Elsevier Health Sciences; 2012.

18. White AA. Clinical biomechanics of the spine. Philadelphia: Lippincott Williams \& Wil; 2006.

19. Boos N, Weissbach S, Rohrbach H, Weiler C, Spratt KF, Nerlich AG. Classification of age-related changes in lumbar intervertebral discs: 2002 Volvo Award in basic science. Spine (Phila Pa 1976) 2002;27:2631-44.

20. Horst M, Brinckmann P. 1980 Volvo award in biomechanics: measurement of the distribution of axial stress on the end-plate of the vertebral body. Spine (Phila Pa 1976) 1981;6:217-32.

21. Kulak RF, Belytschko TB, Schultz AB. Nonlinear behavior of the human intervertebral disc under axial load. J Biomech 1976;9:377-86.

22. Haut RC, Little RW. A constitutive equation for collagen fibers. J Biomech 1972;5:423-30.

23. Markolf KL. Deformation of the thoracolumbar intervertebral joints in response to external loads: a biomechanical study using autopsy material. J Bone Joint Surg Am 1972;54:511-33.

24. Fujiwara A, Tamai K, An HS, et al. The relationship between disc degeneration, facet joint osteoarthritis, and stability of the degenerative lumbar spine. J Spinal Disord 2000;13:444-50.

25. Mulholland RC, Sengupta DK. Rationale, principles and experimental evaluation of the concept of soft stabilization. Eur Spine J 2002;11 Suppl 2:S198-205.

26. Kos J, Hert J, Sevcik P. Meniscoids of the intervertebral joints. Acta Chir Orthop Traumatol Cech 2002; 69:149-57.

27. Tanno I, Murakami G, Oguma H, et al. Morphometry of the lumbar zygapophyseal facet capsule and cartilage with special reference to degenerative osteoarthritic changes: an anatomical study using fresh cadavers of elderly Japanese and Korean subjects. J Orthop Sci 2004;9:468-77.

28. Cyron BM, Hutton WC. The tensile strength of the capsular ligaments of the apophyseal joints. J Anat 1981;132:145-50.

29. Pal GP, Routal RV, Saggu SK. The orientation of the articular facets of the zygapophyseal joints at the cervical and upper thoracic region. J Anat 2001;198:43141.

30. Love TW, Fagan AB, Fraser RD. Degenerative spondylolisthesis. Developmental or acquired? J Bone Joint Surg Br 1999;81:670-4.

31. Yang KH, King AI. Mechanism of facet load trans- mission as a hypothesis for low-back pain. Spine (Phila Pa 1976) 1984;9:557-65.

32. Jinkins JR. Acquired degenerative changes of the intervertebral segments at and suprajacent to the lumbosacral junction: a radioanatomic analysis of the nondiscal structures of the spinal column and perispinal soft tissues. Eur J Radiol 2004;50:134-58.

33. Kalichman L, Hunter DJ. Lumbar facet joint osteoarthritis: a review. Semin Arthritis Rheum 2007;37:6980.

34. Masharawi Y, Rothschild B, Dar G, et al. Facet orientation in the thoracolumbar spine: three-dimensional anatomic and biomechanical analysis. Spine (Phila Pa 1976) 2004;29:1755-63.

35. Lorenz M, Patwardhan A, Vanderby R Jr. Loadbearing characteristics of lumbar facets in normal and surgically altered spinal segments. Spine (Phila Pa 1976) 1983;8:122-30.

36. Tischer T, Aktas T, Milz S, Putz RV. Detailed pathological changes of human lumbar facet joints L1-L5 in elderly individuals. Eur Spine J 2006;15:308-15.

37. Boszczyk BM, Boszczyk AA, Korge A, et al. Immunohistochemical analysis of the extracellular matrix in the posterior capsule of the zygapophysial joints in patients with degenerative L4-5 motion segment instability. J Neurosurg 2003;99:27-33.

38. Lee CK. Accelerated degeneration of the segment adjacent to a lumbar fusion. Spine (Phila Pa 1976) 1988;13:375-7.

39. Hilibrand AS, Robbins M. Adjacent segment degeneration and adjacent segment disease: the consequences of spinal fusion? Spine J 2004;4(6 Suppl): 190S-194S.

40. Hilibrand AS, Carlson GD, Palumbo MA, Jones PK, Bohlman HH. Radiculopathy and myelopathy at segments adjacent to the site of a previous anterior cervical arthrodesis. J Bone Joint Surg Am 1999;81:51928.

41. Eck JC, Humphreys SC, Lim TH, et al. Biomechanical study on the effect of cervical spine fusion on adjacent-level intradiscal pressure and segmental motion. Spine (Phila Pa 1976) 2002;27:2431-4.

42. Lopez-Espina CG, Amirouche F, Havalad V. Multilevel cervical fusion and its effect on disc degeneration and osteophyte formation. Spine (Phila Pa 1976) 2006;31:972-8.

43. Maiman DJ, Kumaresan S, Yoganandan N, Pintar FA. 
Biomechanical effect of anterior cervical spine fusion on adjacent segments. Biomed Mater Eng 1999;9:2738.

44. Matsunaga S, Kabayama S, Yamamoto T, Yone K, Sakou T, Nakanishi K. Strain on intervertebral discs after anterior cervical decompression and fusion. Spine (Phila Pa 1976) 1999;24:670-5.

45. Shono Y, Kaneda K, Abumi K, McAfee PC, Cunning- ham BW. Stability of posterior spinal instrumentation and its effects on adjacent motion segments in the lumbosacral spine. Spine (Phila Pa 1976) 1998;23: 1550-8.

46. Weinhoffer SL, Guyer RD, Herbert M, Griffith SL. Intradiscal pressure measurements above an instrumented fusion: a cadaveric study. Spine (Phila Pa 1976) 1995;20:526-31. 